\title{
Effective Hamiltonian for Excitons with Spin Degrees of Freedom
}

\author{
Jun-ichi Inoue日, Tobias Brandes* \\ Institute of Physics, University of Tokyo, 3-8-1 Komaba, Meguro-ku, Tokyo 153-8902, Japan \\ and Core Research for Evolutional Science and Technology, JST
}

(August 13, 2018)

\begin{abstract}
Starting from the conventional electron-hole Hamiltonian $\mathcal{H}_{e h}$, we derive an effective Hamiltonian $\tilde{\mathcal{H}}_{1 s}$ for $1 s$ excitons with spin degrees of freedom. The Hamiltonian describes optical processes close to the exciton resonance for the case of weak excitation. We show that straightforward bosonization of $\mathcal{H}_{e h}$ does not give the correct form of $\tilde{\mathcal{H}}_{1 s}$, which we obtain by a projection onto the subspace spanned by the $1 s$ excitons. The resulting relaxation and renormalization terms generate an interaction between excitons with opposite spin. Moreover, exciton-exciton repulsive interaction is greatly reduced by the renormalization. The agreement of the present theory with the experiment supports the validity of the description of a fermionic system by bosonic fields in two dimensions.
\end{abstract}

PACS numbers: 71.35.-y, 71.10.-w, 78.66.-w

Typeset using REVTEX 
Properties of a fermionic system can sometimes be described by bosonic fields, by which theoretical analyses may be greatly simplified. The most successful example is the bosonization of one-dimensional $(d=1)$ conductors [1], where success is due to the specific feature of the pair spectrum [2], i.e., the low-energy pair spectra consist of discrete branches in the energy versus momentum plane. For $d \geq 2$ conductors, discrete branches overlap with continuous spectra, hence the bosonization is nontrivial and still in progress [3].

For insulating solids, on the other hand, discrete branches of excitons are separated from the continuum for any $d$. From this point of view, it has been suggested that a useful bosonic theory may be constructed if one focuses on exciton states, even for $d \geq 2$ [4 [7. However, the validity of the bosonic description of excitons is nontrivial, because the binding energy (in, e.g., GaAs) is comparable to other relevant energies [8] excitation, excitons and free electron-hole $(e-h)$ pairs (continuous spectra) will be created. As the excitation intensity (and thus the $e-h$ density) is increased, the fermionic nature of the system becomes more important, and bosonization requires more bosonic fields. Such a strong-excitation regime has been successfully analyzed without the use of bosonization [9 [13]. In a weak-excitation regime, on the other hand, it is expected that the system is well described by a small number of bosonic fields. If this is the case, the bosonic theory will provide a powerful theoretical tool as well as a transparent physical view, as in the case of the $d=1$ conductors [回.

To demonstrate the effectiveness of the bosonized theory, optical experiments may be more convenient than the electron transport experiments, because one can easily produce and detect two or more light beams, and obtain rich information from responses to the multi beams. Moreover, one can easily control the polarization of each individual light beam, which gives more detailed information. Recently, by controlling the polarizations of two light beams, Kuwata-Gonokami et al. demonstrated experimentally that the polaritonpolariton scatterings in a quantum well (QW) in an optical micro cavity are well described by a phenomenological bosonic Hamiltonian in the weak-excitation regime [0.14]. Their experiment strongly indicated the validity of a bosonic description for $d=2$. However, no 
theoretical studies were reported which derive their phenomenological Hamiltonian from a microscopic fermionic theory.

In this letter, we derive an effective Hamiltonian of excitons from the conventional $e-h$ Hamiltonian. In particular, we will show that the derivation of an effective bosonic theory is nontrivial and not straightforward because a direct bosonization does not give correct results. Our calculation and the agreement with the experimental data [5] directly prove the validity and relevance of a bosonic description for $d=2$.

Model - We start from a Hamiltonian $\mathcal{H}_{e h}$ for an interacting electron-hole system in a QW.

$$
\begin{aligned}
\mathcal{H}_{e h}= & \sum_{i} \int \mathrm{d} x \hat{\psi}_{i}^{\dagger}(x)\left(-\frac{\hbar^{2} \nabla^{2}}{2 m_{i}}+E_{i}\right) \hat{\psi}_{i}(x) \\
& +\sum_{i, i^{\prime}} \frac{z_{i} z_{i^{\prime}}}{2} \int \mathrm{d} x \mathrm{~d} x^{\prime} \hat{\psi}_{i}^{\dagger}(x) \hat{\psi}_{i^{\prime}}^{\dagger}\left(x^{\prime}\right) V\left(\mathbf{r}_{i}-\mathbf{r}_{i^{\prime}}^{\prime}\right) \hat{\psi}_{i^{\prime}}\left(x^{\prime}\right) \hat{\psi}_{i}(x) .
\end{aligned}
$$

Here, $V(\mathbf{r})$ denotes the Coulomb potential, which behaves in a $\mathrm{QW}$ of width $L$ as $V(\mathbf{r}) \approx$ $e^{2} / \epsilon r$ for $|\mathbf{r}| \gtrsim L$, where $\epsilon$ is the static dielectric constant, and $V(\mathbf{r}) \approx$ constant for $|\mathbf{r}| \lesssim L$. We simplify the calculation by taking the limit $L \rightarrow 0$ wherever the singularity at $\mathbf{r}=\mathbf{0}$ is irrelevant. In eq. (1), $\hat{\psi}_{e(h)}(x)$ is the field operator of an electron (hole), $\left\{i, i^{\prime}\right\}=\{e, h\}$, $z_{e(h)}=1(-1), x \equiv\left(\mathbf{r}_{e(h)}, J_{e(h)}^{z}\right)$, and $\int \mathrm{d} x \equiv \sum_{J_{i}^{z}} \int \mathrm{d}^{2} r_{i}$. The index $J_{e(h)}^{z}$ denotes the $z-$ component of the total angular momentum (which is a good quantum number), where we take the $z$-axis to be in the direction normal to the QW layers. The $J_{h}^{z}$ is defined as -1 times $J^{z}$ of a valence band electron. In a GaAs QW, e.g., $J_{h}^{z}= \pm 3 / 2$ for a heavy hole, and $J_{e}^{z}= \pm 1 / 2$ [9]. A photon with $J_{p h}^{z}=+1(-1)$ creates an electron-hole pair with $J_{e}^{z}=-1 / 2(+1 / 2)$ for the electron and with $J_{h}^{z}=+3 / 2(-3 / 2)$ for the hole. The exciton states are labelled by indices $\mathbf{q}, \nu$ and $S$, where $\mathbf{q}$ is the momentum of the center-of-mass motion, $\nu$ denotes the set of quantum numbers for the relative motion $\left(\nu=1 s, 2 p_{+}, 2 p_{-}, \cdots\right)$ [9], and $S$ denotes combinations of $J_{e}^{z}$ and $J_{h}^{z}$. Since $S$ is related to the total angular momentum, we call $S$ the "spin" index.

Since all states excited by photons are electrically neutral, we can confine ourselves to the charge-neutral sector. We first transform $\mathcal{H}_{e h}$ to a Hamiltonian $\mathcal{H}$ of interacting excitons. 
We then make a projection onto the subspace spanned by 1s excitons to obtain an effective theory which is described by a renormalized Hamiltonian $\tilde{\mathcal{H}}_{1 s}$ of 1 s excitons and a relaxation $\tilde{\Gamma}$.

Transformation of $\mathcal{H}_{e h}$ into $\mathcal{H}-$ We first rewrite $\mathcal{H}_{\text {eh }}$ as a function of exciton operators 15],

$$
\begin{aligned}
b_{\mathbf{q} \nu S} \equiv & \sum_{J_{e}^{z}, J_{h}^{z}} \int \mathrm{d}^{2} r_{e} \mathrm{~d}^{2} r_{h} \frac{1}{\sqrt{\Omega}} \exp \left(i \mathbf{q} \cdot \frac{m_{e} \mathbf{r}_{e}+m_{h} \mathbf{r}_{h}}{M}\right) \\
& \times \varphi_{\nu}\left(\mathbf{r}_{e}-\mathbf{r}_{h}\right)\left\langle S \mid J_{e}^{z}, J_{h}^{z}\right\rangle \hat{\psi}_{e}\left(\mathbf{r}_{e}, J_{e}^{z}\right) \hat{\psi}_{h}\left(\mathbf{r}_{h}, J_{h}^{z}\right) .
\end{aligned}
$$

Here, $\varphi_{\nu}(\mathbf{r})$ is a wavefunction for the $e$ - $h$ relative motion, $\left\langle S \mid J_{e}^{z}, J_{h}^{z}\right\rangle$ the Clebsch-Gordan (CG) coefficient, $\Omega$ the QW area, and $M \equiv m_{e}+m_{h}$. In the following, we assume $0<m_{e} \ll$ $m_{h}$. To define $S$ uniquely, we choose $S=+,-, \alpha$, and $\beta$, for $\left|J_{e}^{z}, J_{h}^{z}\right\rangle=|-1 / 2,+3 / 2\rangle$, $|+1 / 2,-3 / 2\rangle,|+1 / 2,+3 / 2\rangle$, and $|-1 / 2,-3 / 2\rangle$, respectively, in a GaAs QW 16. An exciton with $S= \pm$ couples to circularly polarized light with $J_{p h}^{z}= \pm 1$, whereas excitons with $S=\alpha$ or $\beta$ are dipole inactive. Then, assuming that the exciton density is low, we regard $b_{\mathbf{q} \nu S}$ 's as genuine boson operators [15] and rewrite $\mathcal{H}_{e h}$ in the charge-neutral sector as $\mathcal{H}_{e h} \rightarrow \mathcal{H}=$ $\mathcal{H}^{0}+\mathcal{H}^{\text {int }}$, where $\mathcal{H}^{0}$ and $\mathcal{H}^{\text {int }}$ are the free and interaction parts of excitons, respectively. Since the transferred momentum in the exciton scattering processes is fairly small, and of the order of the photon momentum, the direct and the double fermionic exchange interactions are negligible and the momentum dependence of the exchange interaction can be omitted. Hence, the general form of $\mathcal{H}^{\text {int }}$ is given by

$$
\begin{aligned}
\mathcal{H}^{i n t}= & \sum_{\mathbf{k} \mathbf{k}^{\prime} \mathbf{q}\{S\}\{\nu\}} \frac{U_{\nu}}{2 \Omega} \sum_{J_{e}^{z} J_{h}^{z} J_{e}^{z \prime} J_{h}^{z \prime}}\left\langle S_{1} \mid J_{e}^{z} J_{h}^{z}\right\rangle\left\langle S_{2} \mid J_{e}^{z \prime} J_{h}^{z \prime}\right\rangle\left\langle S_{3} \mid J_{e}^{z \prime} J_{h}^{z}\right\rangle\left\langle S_{4} \mid J_{e}^{z} J_{h}^{z \prime}\right\rangle \\
& \times b_{\mathbf{k}+\mathbf{q} \nu_{1} S_{1}}^{\dagger} b_{\mathbf{k}^{\prime}-\mathbf{q} \nu_{2} S_{2}} b_{\mathbf{k}^{\prime} \nu_{4} S_{4}} b_{\mathbf{k} \nu_{3} S_{3}} .
\end{aligned}
$$

Using this formula, we express $\mathcal{H}$ as

$$
\mathcal{H}=\mathcal{H}^{0}+\mathcal{H}_{1 s}^{ \pm}+\mathcal{H}_{1 s}^{\prime}+\mathcal{H}_{\text {others }}
$$

where $\mathcal{H}_{1 s}^{ \pm}$and $\mathcal{H}_{1 s}^{\prime}$ include the $\nu=1 s$ operators only, and $\mathcal{H}_{\text {others }}$ denotes the remaining terms. The $\mathcal{H}_{1 s}^{ \pm}$consists only of the operators with $S= \pm$, whereas $\mathcal{H}_{1 s}^{\prime}$ consists of terms of $S=\alpha$ and $\beta$ operators, including cross terms with $S= \pm$ operators: 


$$
\begin{aligned}
\mathcal{H}_{1 s}^{ \pm} & =\frac{U}{2 \Omega} \sum_{S= \pm \mathbf{k k}^{\prime} \mathbf{q}} b_{\mathbf{k}+\mathbf{q} S}^{\dagger} b_{\mathbf{k}^{\prime}-\mathbf{q} S}^{\dagger} b_{\mathbf{k}^{\prime} S} b_{\mathbf{k} S}, \\
\mathcal{H}_{1 s}^{\prime}= & \frac{U}{\Omega} \sum_{\mathbf{k k}^{\prime} \mathbf{q}}\left[\sum _ { S = \alpha , \beta } \left(\frac{1}{2} b_{\mathbf{k}+\mathbf{q} S}^{\dagger} b_{\mathbf{k}^{\prime}-\mathbf{q} S}^{\dagger} b_{\mathbf{k}^{\prime} S} b_{\mathbf{k} S}+b_{\mathbf{k}+\mathbf{q}+}^{\dagger} b_{\mathbf{k}^{\prime}-\mathbf{q} S}^{\dagger} b_{\mathbf{k}^{\prime} S} b_{\mathbf{k}+}\right.\right. \\
& \left.\left.+b_{\mathbf{k}+\mathbf{q}-}^{\dagger} b_{\mathbf{k}^{\prime}-\mathbf{q} S}^{\dagger} b_{\mathbf{k}^{\prime} S} b_{\mathbf{k}-}\right)+\left(b_{\mathbf{k}+\mathbf{q}+}^{\dagger} b_{\mathbf{k}^{\prime}-\mathbf{q}-}^{\dagger} b_{\mathbf{k}^{\prime} \alpha} b_{\mathbf{k} \beta}+\text { H.c. }\right)\right]
\end{aligned}
$$

where we have written $b_{\mathbf{q} 1 s S} \equiv b_{\mathbf{q} S}$, and the effective interaction strength $U \equiv U_{\nu=1 s}$ is expressed as

$$
U=2 \sqrt{\Omega} \sum_{\mathbf{p}, \mathbf{p}^{\prime}} \tilde{V}\left(\mathbf{p}-\mathbf{p}^{\prime}\right)\left[\left|\tilde{\varphi}_{1 s}(\mathbf{p})\right|^{2} \tilde{\varphi}_{1 s}^{*}(\mathbf{p}) \tilde{\varphi}_{1 s}\left(\mathbf{p}^{\prime}\right)-\left|\tilde{\varphi}_{1 s}(\mathbf{p})\right|^{2}\left|\tilde{\varphi}_{1 s}\left(\mathbf{p}^{\prime}\right)\right|^{2}\right]
$$

Here, $\tilde{V}(\mathbf{p})$ and $\tilde{\varphi}_{\nu}(\mathbf{p})$ are the Fourier transforms of $V(\mathbf{r})$ and $\varphi_{\nu}(\mathbf{r})$, respectively. For $d=2$, and in the limit of $L \rightarrow 0$, eq. (7) is evaluated as $U=1.52 a_{0}^{2} E_{x}^{b}$ [17], where $a_{0} \equiv \epsilon \hbar^{2} M / e^{2} m_{e} m_{h}$ and $E_{x}^{b} \equiv 2 e^{2} / \epsilon a_{0}$.

It is tempting to take $\mathcal{H}_{1 s}^{ \pm}+\mathcal{H}_{1 s}^{\prime}$ as an effective interaction Hamiltonian $\mathcal{H}_{1 s}^{\text {eff }}$ for $1 s$ excitons, by simply dropping $\mathcal{H}_{\text {others }}$. However, as we will show below, this is not an appropriate approximation of $\mathcal{H}_{1 s}^{e f f}$, because important terms which describe the interactions between excitons with $S=+$ and with $S=-$ are absent in $\mathcal{H}_{1 s}^{ \pm}+\mathcal{H}_{1 s}^{\prime} 18$.

Projection - The evolution of the density operator $\rho$ in the charge neutral sector obeys the von Neumann equation, $\partial \rho / \partial t=(1 / i \hbar)[\mathcal{H}, \rho]$. To derive the correct $\mathcal{H}_{1 s}^{e f f}$, we make a projection onto the subspace spanned by the 1s excitons. We then obtain the equation for the reduced density operator $\tilde{\rho}_{1 s}$, as $\partial \tilde{\rho}_{1 s} / \partial t=(1 / i \hbar)\left[\tilde{\mathcal{H}}_{1 s}, \tilde{\rho}_{1 s}\right]+\tilde{\Gamma} \tilde{\rho}_{1 s}$. Here, $\tilde{\mathcal{H}}_{1 s}$ describes the unitary evolution of $\tilde{\rho}_{1 s}$, and $\tilde{\Gamma}$ the relaxation operator. We can calculate optical responses using $\tilde{\mathcal{H}}_{1 s}$, neglecting $\tilde{\Gamma}$, when (a) the photon energy is close to the energy of the $1 s$ excitons, (b) the photoexcitation is weak, and (c) relaxation processes do not play an important role during the photon scattering processes. The reason for (a) is that $2 p$ and higher excitons have been projected out. The condition (b) is due to the fact that the deviation from the Bose statistics of operators $b_{\mathbf{q} \nu S}$ and $b_{\mathbf{q} \nu S}^{\dagger}$ becomes non-negligible when the excitation is strong [19]. In the experiment described in ref. [5], the three conditions have been satisfied. To satisfy condition (c), both the resonance of $1 s$ excitons and the strong coupling to the 
radiation field in a high Q micro cavity are crucial [20]. Otherwise, we must take account of $\tilde{\Gamma}$ in the calculations of optical responses.

The renormalization procedure yields [2]

$$
\tilde{\mathcal{H}}_{1 s}=\tilde{\mathcal{H}}_{1 s}^{0}+\tilde{\mathcal{H}}_{1 s}^{ \pm}+\tilde{\mathcal{H}}_{1 s}^{\prime}
$$

where $\tilde{\mathcal{H}}_{1 s}^{0}$ is the Hamiltonian of free $1 s$ excitons, and $\tilde{\mathcal{H}}_{1 s}^{ \pm}$and $\tilde{\mathcal{H}}_{1 s}^{\prime}$ include the $\nu=1 s$ operators only. The $\tilde{\mathcal{H}}_{1 s}^{ \pm}$consists only of operators with $S= \pm$, whereas $\tilde{\mathcal{H}}_{1 s}^{\prime}$ consists of terms of $S=\alpha$ and $\beta$ operators, including cross terms with $S= \pm$ operators. Since $\tilde{\mathcal{H}}_{1 s}^{\prime}$ includes dipole inactive $1 s$ excitons $(S=\alpha, \beta)$, it does not contribute to the optical response in its lowest order [14,22]. The most important terms are therefore included in $\tilde{\mathcal{H}}_{1 s}^{ \pm}$, which is evaluated, to the second order in the exciton-exciton interactions, as

$$
\tilde{\mathcal{H}}_{1 s}^{ \pm}=\frac{U-U^{\prime}}{2 \Omega} \sum_{S= \pm \mathbf{k k}^{\prime} \mathbf{q}} b_{\mathbf{k}+\mathbf{q} S}^{\dagger} b_{\mathbf{k}^{\prime}-\mathbf{q} S}^{\dagger} b_{\mathbf{k}^{\prime} S} b_{\mathbf{k} S}-\frac{U^{\prime}}{\Omega} \sum_{\mathbf{k k}^{\prime} \mathbf{q}} b_{\mathbf{k}+\mathbf{q}+}^{\dagger} b_{\mathbf{k}^{\prime}-\mathbf{q}-}^{\dagger} b_{\mathbf{k}^{\prime}-} b_{\mathbf{k}+},
$$

where $U^{\prime}$ is a positive constant which arises from the renormalization of higher exciton states $\left(\nu=2 p_{+}, 2 p_{-}, \cdots\right):$

$$
\begin{aligned}
U^{\prime}=\Omega & \sum_{\mathbf{K}, \nu \neq 1 s} \frac{1}{2\left(E_{\nu}+\mathbf{K}^{2} / 2 M\right)-2 E_{1 s}} \mid \sum_{\mathbf{p}, \mathbf{p}^{\prime}} \tilde{V}\left(\mathbf{p}-\mathbf{p}^{\prime}+\mathbf{K}\right)\left[-\tilde{\varphi}_{1 s}^{*}(\mathbf{p}) \tilde{\varphi}_{1 s}^{*}\left(\mathbf{p}^{\prime}\right) \tilde{\varphi}_{\nu}(\mathbf{p}) \tilde{\varphi}_{\nu}\left(\mathbf{p}^{\prime}\right)\right. \\
& \left.+2 \tilde{\varphi}_{1 s}^{*}(\mathbf{p}) \tilde{\varphi}_{1 s}^{*}(\mathbf{p}-\mathbf{K}) \tilde{\varphi}_{\nu}(\mathbf{p}) \tilde{\varphi}_{\nu}\left(\mathbf{p}^{\prime}\right)-\tilde{\varphi}_{1 s}^{*}(\mathbf{p}) \tilde{\varphi}_{1 s}^{*}\left(\mathbf{p}^{\prime}\right) \tilde{\varphi}_{\nu}(\mathbf{p}-\mathbf{K}) \tilde{\varphi}_{\nu}\left(\mathbf{p}^{\prime}+\mathbf{K}\right)\right]\left.\right|^{2}
\end{aligned}
$$

Comparing the right-hand side of eq. (9) with that of eq. (5), we find that the coefficient of the first term is renormalized as $U \rightarrow U-U^{\prime}$, and that a second term is generated which leads to an interaction between the $S=+$ and - excitons. That is, the renormalization of higher exciton states results in the renormalized Hamiltonian $\tilde{\mathcal{H}}_{1 s}^{ \pm}$, which differs, both quantitatively and qualitatively, from the bare Hamiltonian $\mathcal{H}_{1 s}^{ \pm}$.

We argue that the correct form of the effective Hamiltonian for $1 s$ excitons is the renormalized one, i.e., $\mathcal{H}_{1 s}^{\text {eff }}=\tilde{\mathcal{H}}_{1 s}^{ \pm}+\tilde{\mathcal{H}}_{1 s}^{\prime}$. In fact, the interaction between the $S=+$ and excitons in $\tilde{\mathcal{H}}_{1 s}^{ \pm}$, which is absent in $\mathcal{H}_{1 s}$, has been clearly observed experimentally in refs. [5] and [23]. Kuwata-Gonokami et al. [5] expressed this interaction as an interaction term 
(whose coupling constant is $W$ ) in the phenomenological Hamiltonian, which also has the interaction term (whose coupling constant is $R$ ) of excitons with parallel spins. The phenomenological Hamiltonian has the same form as $\tilde{\mathcal{H}}_{1 s}^{ \pm}$, the dipole active part of $\tilde{\mathcal{H}}_{1 s}$. This is quite reasonable because the other part $\tilde{\mathcal{H}}_{1 s}^{\prime}$, which is dipole inactive, should be invisible in low-order optical experiments [22]. We can, therefore, identify the parameters $R$ and $W$ of the phenomenological Hamiltonian [5] as

$$
\begin{aligned}
R & =\left(1.52 a_{0}^{2} E_{x}^{b}-U^{\prime}\right) /(2 \Omega), \\
W & =-U^{\prime} / \Omega .
\end{aligned}
$$

The value of $U^{\prime}$, as given by eq. (10), depends on the material parameters such as $M$ and $\epsilon$, and hence it is different for different materials. It also depends on the QW parameter $L$. Moreover, when imperfections in the QW are non-negligible, the formula for $U^{\prime}$ should be modified accordingly. Therefore, even for the same material, the values of $R$ and $W$ could vary slightly from sample to sample, which seems to be consistent with recent experimental results [20]. Note, however, that the existence of both terms of $\tilde{\mathcal{H}}_{1 s}^{ \pm}$is independent of such details.

We here estimate the typical value of $U^{\prime}$ as follows. The $\mathbf{K}$-summation in eq. (10) is cutoff for $K \gtrsim C_{L} / L$ (through the cutoff of $\tilde{V}$ ) and/or for $K \gtrsim C_{a_{0}} / a_{0}$ (through $\tilde{\varphi}_{\nu}$ ), where $C_{L}$ and $C_{a_{0}}$ are cutoff parameters of the order of unity. For the case of the QW sample of ref. [5], $L \approx a_{0}$, hence we may cutoff the $\mathbf{K}$-summation for $K \gtrsim C / a_{0}$, where $C$ is of the order of unity. For the $\nu$ summation, we may consider $\nu=2 p_{ \pm}$states only, because higher exciton states give much smaller overlap integrals. These approximations yield $U^{\prime} \approx 16.5 C^{2} a_{0}^{2} E_{x}^{b}$. On the other hand, ref. [5] reported the ratio $R: W$ as $1:-15$. From eqs. (11) and (12), we find that this ratio is reproduced by the present theory when the cutoff parameter $C \sim 0.3$, which is consistent with the requirement that $C$ is of the order of unity. Considering that the values of $R$ and $W$ vary slightly from sample to sample [20], the agreement seems satisfactory. Note that such a small value of $R$ is due to the renormalization of $U \rightarrow U-U^{\prime}$. Once the agreement of $\tilde{\mathcal{H}}_{1 s}^{ \pm}$with the phenomenological Hamiltonian is thus established, the 
agreement with the experiment follows. That is, lowest-order perturbational calculations for the polariton-polariton scattering amplitudes agree with the experiment [5, 14].

Discussions and remarks - It was conjectured [5] that a "biexciton effect" would be the origin of the " $W$ term", the interaction between $S=+$ and - excitons. However, this argument is misleading. The biexciton state is formed essentially from the mixing of two $1 s$ states having different centers. For examples, in the case of a hydrogen molecule, the mixing yields the bonding and antibonding states, $(1 / \sqrt{2})\left(c_{1 \uparrow}^{\dagger} c_{2 \downarrow}^{\dagger} \pm c_{1 \downarrow}^{\dagger} c_{2 \uparrow}^{\dagger}\right) h_{1 \sigma}^{\dagger} h_{2 \sigma^{\prime}}^{\dagger}|0\rangle$. Here, $c_{1(2)}^{\dagger}$ creates an electron in the $1 s$ state located at nucleus $1(2)$, and $h_{1(2)}^{\dagger}$ creates the nucleus. In the case of excitons with $J_{e}^{z}= \pm 1 / 2$ and $J_{h}^{z}= \pm 3 / 2$ [16], the corresponding states are $(1 / \sqrt{2})\left[b_{+}^{\dagger} b_{-}^{\dagger} \pm b_{\alpha}^{\dagger} b_{\beta}^{\dagger}\right]|0\rangle$, where we have not shown the $\mathbf{k}$-dependence in order to focus on the $S$-dependence. The bonding state ( - sign for a positive coupling constant) has a lower energy and is called a biexciton. This energy splitting between the bonding and antibonding states is induced by the interaction of the form of $b_{+}^{\dagger} b_{-}^{\dagger} b_{\alpha} b_{\beta}+H . c .$, which is included in $\tilde{\mathcal{H}}_{1 s}^{\prime}$ (or, before the renormalization, in $\mathcal{H}_{1 s}^{\prime}$ of eq. (6)) [24]. On the other hand, the $W$ term lowers the energies of both states by the same amount, hence does not play a central role in the formation of the biexciton state. The most important effect of the $W$ term is to lower the energy of $b_{+}^{\dagger} b_{-}^{\dagger}|0\rangle$, relative to those of $b_{+}^{\dagger} b_{+}^{\dagger}|0\rangle$ and $b_{-}^{\dagger} b_{-}^{\dagger}|0\rangle$, and this effect was detected experimentally [5]. In short, in the framework of the present theory, $\tilde{\mathcal{H}}_{1 s}^{\prime}$ lowers the energy of the bonding (biexciton) state relative to that of the antibonding state, and thus is crucial for the formation of the biexciton state, whereas the $W$ term lowers the energy of both bonding and antibonding states.

Note that $\tilde{\mathcal{H}}_{1 s}$ is not positive definite to the fourth order in the exciton operators. The stability of the system should be preserved by higher order terms. In general situations, properties of a system described by such a Hamiltonian should not be analyzed by a perturbation theory based on the vacuum of the free part. Nevertheless, we can use such a perturbation theory in our case, because our exciton theory has the built-in constraint that the ground state is the state with no excitons, i.e., the vacuum of $\tilde{\mathcal{H}}_{1 s}^{0}$. The effective Hamiltonian $\tilde{\mathcal{H}}_{1 s}$ together with this constraint constitutes a consistent theory, which justifies 
the low-order perturbation theory based on the given vacuum, if the optical excitation is sufficiently weak.

We have used a low-order perturbation theory to derive $\tilde{\mathcal{H}}_{1 s}$. However, this does not imply a total neglect of higher order terms, because we have calculated a Hamiltonian rather than observables. In fact, a systematic summing up of higher order terms is already incorporated in our theory if one calculates higher order scattering amplitudes, e.g., by writing the Bethe-Salpeter equation and using $\tilde{\mathcal{H}}_{1 s}$.

Finally, we discuss the relation between the fermionic theories [9] and our bosonic theory. The Hartree-Fock (HF) factorization treatment of the semiconductor Bloch equations [10 can not produce the interaction between the $S=+$ and - excitons. The HF theory, therefore, corresponds to $\mathcal{H}_{1 s}^{ \pm}$, eq. (5). It was argued in refs. [11]13] that the interactions of an exciton with higher states (including free carriers) are important, and that the interactions result in the energy shift, the excitation-induced dephasing (EID), and the "biexcitonic correlations". In the bosonic theory in the form of eq. (ㅍ) , these effects are included in $\mathcal{H}_{1 s}^{\prime}$ and $\mathcal{H}_{\text {others }}$. After the projection is made, the relation is roughly as follows. The renormalized Hamiltonian $\tilde{\mathcal{H}}_{1 s}^{ \pm}$, eq. (9), would include the HF term and a part of the "biexcitonic correlation." The EID may be described by both $\tilde{\Gamma}$ and $\tilde{\mathcal{H}}_{1 s}^{\prime}$. Another part of the "biexcitonic correlation" would also be included in $\tilde{\mathcal{H}}_{1 s}^{\prime}$. The present theory thus helps to bridge the gap between the bosonic theories [47] and the fermionic theories [9 13] of $e-h$ systems. However, more detailed comparisons will be a subject of future studies.

Summary and Conclusions - In this letter, starting from the conventional electron-hole Hamiltonian (eq. (1)), we have derived the effective Hamiltonian $\tilde{\mathcal{H}}_{1 s}$ for the $1 s$ excitons with the spin degrees of freedom using the bosonic exciton operators in two dimensions (eq. $(2))$. It is found that the renormalization associated with the projection onto the $1 s$ exciton space is crucial, which leads to the generation of the attractive interaction between excitons with opposite spins (eq. (12)), and to the large reduction of the repulsive interaction between excitons with parallel spins (eq. (11)). Such a drastic modification of the interactions was absent in the previous theory without the renormalization procedure. The present theory 
is valid for systems that satisfy the following conditions: (i) excitation is weak, (ii) the $1 s$ excitons play a crucial role, and (iii) the exciton relaxation process is less important due to, for example, the micro cavity of a high Q-value. This effective Hamiltonian provides the microscopic foundation of the phenomenology proposed in ref. [5]. The agreement of the present theory with the experiment supports the validity of the description of a fermionic system by bosonic fields in two dimensions.

Helpful discussions with Professor Kuwata-Gonokami and Dr. Suzuura are acknowledged. 


\section{REFERENCES}

* Electronic address: inoue@ASone.c.u-tokyo.ac.jp

** Present address: 1. Inst. Theor. Physik, Jungiusstr. 9, D-20355 Hamburg, Germany

[1] M. Stone: Bosonization (World Scientific, Singapore, 1994).

[2] F. D. M. Haldane: J. Phys. C14 (1981) 170.

[3] P. Kopietz: Bosonization of Interacting Fermions in Arbitrary Dimensions (Springer, Berlin, 1997).

[4] E. Hanamura and H. Haug: Phys. Rep. 33 (1977) 209.

[5] M. Kuwata-Gonokami, S. Inouye, H. Suzuura, M. Shirane, R. Shimano, T. Someya and H. Sakaki, Phys. Rev. Lett. 79, 1341 (1997).

[6] V. M. Axt and S. Mukamel: Rev. Mod. Phys. 70 (1998) 145.

[7] A. L. Ivanov, H. Haug and L. V. Keldysh: Phys. Rep. 296 (1998) 237 and references therein.

[8] This should be contrasted with liquid Helium 4: there is no doubt that an Helium 4 atom can be described by a boson operator when the superfluidity is studied, because its nuclear binding energy is much larger than typical energy scales of superfluid experiments.

[9] See, for example, H. Haug and S. W. Koch: Quantum Theory of the Optical and Electric Properties of Semiconductors (World Scientific, Singapore, 1994) 3rd ed.

[10] M. Lindberg and S. W. Koch: Phys. Rev. B38 (1988) 3342.

[11] Y. Z. Hu, R. Binder, S. W. Koch, S. T. Cundiff, H. Wang and D. G. Steel, Phys. Rev. B49, 14382 (1994).

[12] T. Rappen, U. B. Peter, M. Wegener and W. Schäfer: Phys. Rev. B49 (1994) 10774. 
[13] W. Schäfer, D. S. Kim, J. Shah, T. C. Damen, J. E. Cunningham, K. W. Goossen, L. N. Pfeiffer and K. Köhler, Phys. Rev. B53, 16429 (1996).

[14] It should be stressed that fairly good agreement is obtained in ref. [5] between the experimental results and the lowest-order calculations based on the phenomenological Hamiltonian.

[15] H. Haug and S. Schmitt-Rink: Prog. Quant. Electr. 9 (1984) 3.

[16] We have obtained a similar result for the case of $J_{h}= \pm 1 / 2$ [21].

[17] S. Schmitt-Rink, D. S. Chemla and D. A. B. Miller: Adv. Phys.39 (1989) 89.

[18] Ivanov and Haug obtained this interaction without performing the projection (Phys. Rev. B48 (1993) 1490). However, they dropped CG coefficients of eq. (3), and determined the spin dependence using another theory of a four-body problem. Hence their derivation is not fully microscopic.

[19] M. Hawton and D. Nelson: Phys. Rev. B57 (1998) 4000.

[20] M. Kuwata-Gonokami: private communication.

[21] J. Inoue, T. Brandes and A. Shimizu (unpublished).

[22] However, $\tilde{\mathcal{H}}_{1 s}^{\prime}$, of course, becomes important when one measures optical processes in which higher-order terms are involved.

[23] K. Bott, O. Heller, D. Bennhardt, S. T. Cundiff, P. Thomas, E. J. Mayer, G. O. Smith, R. Eccleston, J. Kuhl, and K. Ploog, Phys. Rev. B48, 17418 (1993).

[24] A typical bound state in an interacting boson model is the eigenstate $|\phi\rangle=\left(a^{\dagger} b^{\dagger}-\right.$ $\left.\operatorname{sign}(g) c^{\dagger} d^{\dagger}\right)|0\rangle$ of $\mathcal{H}=\omega\left(a^{\dagger} a+b^{\dagger} b+c^{\dagger} c+d^{\dagger} d\right)+g\left(a^{\dagger} b^{\dagger} c d+d^{\dagger} c^{\dagger} b a\right)$. The eigenenergy is $2 \omega-|g|$. Note that the existence of the bound state is independent of the sign of the interaction $g$. 